\title{
THE
}

\section{Effect of cilostazol in treating diabetes-associated microvascular complications}

Nicole J. Asal

University of Rhode Island, nicole_asal@uri.edu

A. Wojciak

University of Rhode Island

Follow this and additional works at: https://digitalcommons.uri.edu/php_facpubs

The University of Rhode Island Faculty have made this article openly available.

Please let us know how Open Access to this research benefits you.

This is a pre-publication author manuscript of the final, published article.

Terms of Use

This article is made available under the terms and conditions applicable towards Open Access

Policy Articles, as set forth in our Terms of Use.

\section{Citation/Publisher Attribution}

Asal, N.J., \& Wojciak, K.A. (2017). Effect of cilostazol in treating diabetes-associated microvascular complications. Endocrine, 56(2), 240-244. doi: 10.1007/s12020-017-1279-4

Available at: https://doi.org/10.1007/s12020-017-1279-4 


\title{
Effect of cilostazol in treating diabetes-associated microvascular complications
}

\author{
Nicole J. Asal ${ }^{1} \cdot$ Karolina A. Wojciak ${ }^{1}$
}

\begin{abstract}
Purpose Cilostazol (Pletal), a phosphodiesterase-3 inhi- bitor, was approved in the United States in 1999 to reduce symptoms of intermittent claudication. Cyclic adenosine monophosphate levels increase from inhibition of phos- phodiesterase resulting in anti-platelet, anti-inflammatory, and vasodilatory effects. Diabetes mellitus is a chronic disease that causes endothelial and platelet dysfunction leading to both microvascular and macrovascular compli- cations. This mini-review highlights the emerging evidence suggesting benefits of using cilostazol in treating micro- vascular complications associated with diabetes mellitus. Methods A review of literature was conducted using PubMed and Embase databases focusing on cilostazol use in diabetes mellitus. Results Cilostazol demonstrated renoprotective effects in patients with diabetic nephropathy by reducing serum soluble adhesion molecule-1 and monocyte chemoattractant protein-1. Cilostazol's anti-inflammatory actions predictably attenuate glomerular damage from increased leukocyte adherence. Additionally, cilostazol delayed renal dysfunc- tion secondary to type 2 diabetes mellitus as albuminuria was reduced most likely resulting from inhibition of nuclear factor kappa-induced inflammatory and endothelial mar- kers. Cilostazol's antiinflammatory actions in addition to its vasodilatory actions relieved retinal hypoxia and decreased excessive production of retinal blood vessels suggesting benefit in diabetic retinopathy. Cilostazol did not improve
\end{abstract}

$\approx$ Nicole J. Asal nicole_asal@uri.edu

${ }^{1}$ Department of Pharmacy Practice, College of Pharmacy, University of Rhode Island, Kingston, Rhode Island, USA 
neuropathy symptom scores signifying that it may not be as beneficial in patients with diabetic peripheral neuropathy without diabetic nephropathy or diabetic retinopathy.

Conclusions Cilostazol's pleiotropic effects may be bene- ficial in patients with type 2 diabetes mellitus and diabetic nephropathy. Additional, larger studies need to be con- ducted to assess the benefits and risks of using cilostazol as an alternative agent in treating patients with diabetic microvascular complications.

Keywords Cilostazol $\cdot$ Diabetic nephropathy $\cdot$ Diabetic retinopathy $\cdot$ Diabetic neuropathy

\section{Introduction}

Cilostazol (Pletal), a phosphodiesterase-3 inhibitor, was approved in the United States in 1999 to reduce symptoms of intermittent claudication. Prior to its approval in the United States market, cilostazol was registered for 10 years in Japan [1]. Its off-label uses include secondary prevention of cerebrovascular accident, coronary stent stenosis, and percutaneous coronary intervention. Patients with diabetes mellitus have elevated platelet reactivity and cilostazol, combined with aspirin and clopidogrel, has been found to be more effective in secondary prevention of cerebral infarction than aspirin and clopidogrel alone [2]. Compared with other antiplatelet agents, cilostazol was found to inhibit carotid intima-media thickness progression in patients with type 2 diabetes mellitus, and therefore may be helpful in controlling carotid atherosclerosis [3].

Phosphodiesterase (PDE) enzymes are grouped into eleven broad families, PDE-1 to PDE-11, based on their various properties and structures. PDE-3 inhibitors can be further subdivided into PDE-3As and PDE3Bs. PDE-3As are found in platelets, vascular smooth muscle cells, and cardiac myocytes, while PDE-3Bs are found in adipocytes, hepatocytes, pancreatic $\beta$ cells, and macrophages [4]. By inhibiting PDEs, cyclic adenosine monophosphate levels increase resulting in pleiotropic effects such as anti-platelet, antiinflammatory, and vasodilatory effects.

Diabetes mellitus is a chronic disease that causes endo- thelial and platelet dysfunction [5]. Endothelial dysfunction develops with disease progression leading to both macro- vascular and microvascular complications. Reduced angio- genesis in the brain, heart, and legs leads to macrovascular complications, whereas excessive angiogenesis in the eye, kidney, and nerves causes microvascular complications [6]. Nontraditional uses of cilostazol have been explored as PDE-3s are found extensively throughout the body. This mini-review highlights the emerging evidence suggesting benefits of using cilostazol in treating microvascular com- plications associated with diabetes mellitus.

\section{Diabetic nephropathy}

Diabetic nephropathy can cause end-stage renal disease and is characterized by proteinuria with early increases in urinary albumin levels $[7,8]$. Reactive oxygen species, induced by hyperglycemia, cause both direct and indirect endothelial damage by modifying extracellular matrix pro- teins and by escalating the inflammatory response $[9,10]$. Nuclear factor kappa $B$ is triggered by reactive oxygen species and induces monocyte chemotactic protein-1 (MCP-1), a pro-inflammatory chemokine, which contributes to renal deterioration by recruiting macrophages [11-14].

In a randomized, placebo-controlled trial, cilostazol was evaluated in 90 Taiwanese patients with diabetic nephro- pathy and peripheral arterial occlusion disease [15]. The effects of cilostazol in delaying renal dysfunction secondary to type 2 diabetes mellitus were assessed through changes in urinary microalbuminuria (MAU) and albumin-to-creatinine ratio (ACR). Only patients with type 2 diabetes mellitus with an A1C between 7.0 and $12.0 \%$ were included in the study and were given either oral cilostazol $100 \mathrm{mg}$ twice daily $(n$ 
$=45)$ or placebo $(n=45)$ for 52 weeks. Partici- pants were subdivided into two groups based on baseline values: MAU (ACR 30-300 $\mu \mathrm{g} / \mathrm{mg}$ ) or macroalbuminuria (ACR $>300 \mu \mathrm{g} / \mathrm{mg}$ ). The average age, duration of diabetes, and baseline A1C values were similar between groups. Patients in the cilostazol-treated group had a greater body- mass index, were more likely to smoke, and more likely to take statins, ACE-Is/ARBs, and aspirin. After 52 weeks of treatment, cilostazol reduced albuminuria $(p=0.024)$ and subsequently reduced the ACR $(p=0.02)$ compared with placebo; however, the values were only marginally reduced in patients with MAU $(p=0.06)$ after subdivision. A regression analysis found that changes in albuminuria and ACR were associated with notable changes in inflammatory and endothelial markers, suggesting that cilostazol exhibits protective effects through its anti-inflammatory actions. Treatment with cilostazol significantly reduced the inflam- matory marker highly-sensitive C-reactive protein $(p=0.03)$, endothelial markers E-selectin $(p<$ $0.001)$ and solu- ble vascular cell adhesion molecule- $1(p<0.001)$. Results of the study suggest that renal deterioration resulting from an upregulated immune response mediated by NF-KB-induced inflammatory and endothelial markers may be suppressed with cilostazol by inhibiting NF-kB [15-17]. Additionally, as supported by another study, cilostazol may ameliorate peripheral arterial occlusion disease, a macro- vascular complication of diabetes mellitus, in patients with type 2 diabetes mellitus by attenuating pro-inflammatory markers [18].

The renoprotective effects of cilostazol in diabetic nephropathy were demonstrated in another study [19]. Jiao et al. measured changes in serum soluble adhesion molecule-1 (sICAM-1) and MCP-1 levels in patients with type 2 diabetes mellitus with and without early diabetic nephropathy. Early nephropathy was defined as a urinary albumin excretion rate level between 30 and $300 \mathrm{mg} / 24 \mathrm{~h}$. Participants were divided into three groups with the first group (Group A) consisting of 20 subjects without protei- nuria. Forty participants with early diabetic nephropathy were evenly divided into the second (Group B) and third groups (Group C). Subjects in Group B received cilostazol $100 \mathrm{mg}$ twice daily, while subjects in Group C received placebo. Treatment lasted for 6 months and participants were not to take aspirin, other antiplatelet agents, anticoagulants, or vasodilators during the study period. Prior to the start of the study, the sICAM-1 levels in the three groups were: Group A $214.5+94.6 \mathrm{ng} / \mathrm{mL}$, Group B 450.0

$+100.7 \mathrm{ng} / \mathrm{mL}$, and Group C $422.0+422.9 \mathrm{ng} / \mathrm{mL}$.

Patients with early diabetic nephropathy had higher baseline levels of sICAM-1, suggesting that the occurrence of dia- betic nephropathy is associated with increased levels of sICAM-1 causing glomerular damage from increased leu- kocyte adherence [20, 21]. After treatment with cilostazol, sICAM-1, MCP-1, and urinary albumin excretion rate decreased significantly (sICAM-1 285.1 +94.4, $p<0.01$ ) compared with treatment with placebo (sICAM-1 412.3 + 89.6). Additional measures in the study included blood pressure, liver and kidney function, and $\mathrm{HbA1c}$; however, no significant changes were observed after treatment with cilostazol.

Together, the results from both studies demonstrate that the proposed mechanism of action by which cilostazol ameliorates the progression of renal dysfunction in patients with diabetic nephropathy is through reduced inflammation. 
Diabetes-induced retinal neurodegeneration results from increased vascular permeability and disordered retinal angiogenesis [7]. Glucose toxicity causes endothelial dys- function, which increases permeability allowing circulating macrophages and pro-inflammatory cytokines to enter and cause neuronal damage [22]. Both hyperglycemia and hypoxia damage the endothelium by triggering cells to produce signal proteins such as vascular endothelial growth factor (VEGF) [23, 24]. Current treatments for diabetic retinopathy focus on inhibiting VEGF since overproduction results in disordered angiogenesis [25]. However, reducing rather than blocking VEGF may be an alternative strategy since VEGF initially protects cells by re-establishing oxy- gen delivery [25].

In a preclinical study performed in rats, the effects of oral cilostazol $(30 \mathrm{mg} / \mathrm{kg})$ on retinal neurodegeneration were compared with $0.9 \%$ saline solution [24]. Diabetic Otsuka Long-Evans Tokushima Fatty (OLETF) rats and matched nondiabetic Long-Evans Tokushima Otsuka (LETO) control rats were treated daily for 15 weeks with either cilostazol or placebo. Glial fibrillary acidic protein immunostaining and VEGF immunoreactivity demonstrated that cilostazol treat- ment improved retinal stress and ischemia, respectively. Retinal glial fibrillary acidic protein and VEGF protein levels decreased in both OLETF and LETO rats with cilostazol treatment; however, levels decreased significantly only in OLETF rats. Cilostazol was also able to significantly reduce retinal ganglion cell death in OLETF diabetic rats, suggesting that it may protect against diabetic retinopathy.

As demonstrated in the study, cilostazol's vasodilatory properties relieve hypoxia by improving ischemia and its antiinflammatory properties reduce excessive production of blood vessels by decreasing VEGF expression. Human studies need to be conducted to support these results to further evaluate cilostazol's effect in protecting against neuronal damage and prevent vision loss in patients with diabetes.

\section{Diabetic neuropathy}

In a small pilot study, cilostazol (100, $200 \mathrm{mg} / \mathrm{day})$ was compared with placebo to evaluate changes in neuropathy symptom scores (NSS) in Filipino patients with type 2 diabetes mellitus and with diabetic peripheral neuropathy [26]. Neuropathy was diagnosed through the accordance of two neurologists and was assessed by comparing patient- reported NSS taken at baseline, week 4, week 8, and week

12. Additionally, walking distance and walking speed were assessed using a Walking Impairment Questionnaire. Patients were divided into three groups: 15 patients treated with placebo, 16 patients treated with cilostazol $100 \mathrm{mg} / \mathrm{day}$, 
and 16 patients treated with cilostazol $200 \mathrm{mg} /$ day. Patients included in the study were diagnosed with type 2 diabetes mellitus more than 3 months prior to start of the trial, were in good glycemic control ( $\mathrm{HbA} 1 \mathrm{c} \leq 8 \%$ ), and were maintained on their diabetes medications during the study period. The mean age between treatment groups was 56 years with a greater proportion of women in each treatment group. Patients in the placebo-treated group were on average hea- vier, while patients in the low-dose cilostazol treatment group were diagnosed with diabetes for a longer period of time. Although overall NSS did improve from baseline to

12 weeks, there were no significant differences in NSS between treatment groups (at 12 weeks, $p=0.333$ ). Walking speed improved significantly ( $p=0.028$ ) with low-dose cilostazol treatment suggesting improved peripheral blood flow. The most common reported adverse events at week 4 were headaches (12 patients) and palpitations ( 8 patients); however, these side effects were not reported at week 12 of the study. Out of the 12 patients that reported headaches, three were receiving placebo, four were receiving low-dose cilostazol, and five were receiving high-dose cilostazol. Results from this study did not demonstrate significant improvement in relieving the symptoms of diabetic neuro- pathy with cilostazol therapy. However, increased walking speed in the low dose-treated cilostazol group suggests potential benefit in patients with diabetes.

\section{Adverse reactions}

In the trials reviewed above, cilostazol did not change body mass index, hemoglobin A1c, fasting glucose, and anklebrachial index $[8,15,24,26]$. Since blood glucose levels were not improved, good glycemic control in patients with diabetes is essential and is likely the rationale for appro- priate glycemic control as an inclusion criterion [15, 26]. In the Tang et al. study, two patients withdrew due to severe dizziness in the cilostazol-treated group, while in the Rosales et al. study patients treated with cilostazol experi- enced more headaches and palpitations, however, these side effects disappeared after week 8 [26]. The risks and benefits of initiating and continuing cilostazol should be evaluated for each patient especially in patients with a history of angina, cardiac arrhythmias, and uncontrolled hypertension [27]. Dizziness, headaches, and palpitations are common adverse reactions related to cilostazol as well as are diar- rhea, pharyngitis, and rhinitis [27]. Furthermore, because of cilostazol's potential to induce palpitations and tachyar- rhythmia, its use is contraindicated in patients with con- gestive heart failure [27]. Serious adverse reactions include atrial fibrillation, cardiac arrest, myocardial infarction, and ventricular tachycardia, as well as bleeding and hemorrha- ging [27]. In a case report including three patients, a potential relationship between cilostazol-induced 
tachycardia and hyperglycemia was observed [28]. All three patients had a past medical history of type 2 diabetes and were admitted for cerebral infarction. Cilostazol was initiated in each patient to prevent a subsequent cerebral infarction. All three patients had an elevated HbA1c and included an HbA1c of 10.7, 10.3, and 9.3\% upon admission for patients 1, 2, and 3, respectively. Two out of the three patients were switched from cilostazol to clopidogrel as their heart rates increased following cilostazol initiation. Notable decreases in heart rate and blood glucose were noted in both patients following drug substitution. Patient 2 benefited from an increase in heart rate as he had sinus bradycardia with first degree atrioventricular block and therefore continued cilos- tazol. The effects of cilostazol on glycemic control require further investigation as the studies above did not show a change in fasting glucose and hemoglobin A1c. However, all patients included in the studies had good glycemic control unlike the three patients included in the case report. For patients without good glycemic control, cilostazol initiation may not be appropriate and if initiated, then close monitoring to adjust diabetes medications may be necessary.

\section{Conclusion}

Cilostazol shows potential benefit in treating microvascular diabetic complications such a diabetic nephropathy. It is important to keep in mind that most of the clinical studies evaluating cilostazol's effects were conducted primarily in Asian populations with a small number of subjects. While evidence exists, further studies in more diverse, human populations need to be conducted to fully evaluate cilosta- zol's therapeutic potential in ameliorating diabetic micro- vascular complications.

Compliance with ethical standards

Conflict of interest The authors declare that they have no conflict of interest.

\section{References}

1. F. Gotoh, H. Tohgi, S. Hirai, et al., Cilostazol stroke prevention study: A placebo-controlled double-blind trial for secondary pre- vention of cerebral infarction. J Stroke Cerebrovasc Dis, 9, 147-157 (2000)

2. D.J. Angiolillo, P. Capranzano, J.L. Ferreiro et al., Impact of adjunctive cilostazol therapy on platelet function profiles in patients with and without diabetes mellitus on aspirin and clopi- dogrel therapy. Thromb. Haemost. 106, 253-262 (2011)

3. J.H. Huh, H. Seok, B.W. Lee, E.S. Kang, H.C. Lee, B.S. Cha, Effect of cilostazol on carotid intima-media thickness in type 2 diabetic patients without cardiovascular event. Endocrine 47, 138-145 (2014)

4. Y. Shakur, L.S. Holst, T.R. Landstrom, M. Movsesian, E. Degerman, V. Manganiello, Regulation and function of the cyclic 
nucleotide phosphodiesterase (PDE3) gene family. Prog. Nucleic Acid Res. Mol. Biol. 66, 241-277 (2001)

5. A. Schäfer, J. Bauersachs, Endothelial dysfunction, impaired endogenous platelet inhibition and platelet activation in diabetes and atherosclerosis. Curr. Vasc. Pharmacol. 6, 52-60 (2008)

6. F. Biscetti, G. Straface, D. Pitocco, F. Zaccardi, G. Ghirlanda, A. Flex, Peroxisome proliferator-activated receptors and angiogen- esis. Nutr. Metab. Cardiovasc. Dis. 19, 751-759 (2009)

7. G. Jerums, S. Panagiotopoulos, E. Premaratne, R.J. MacIsaac, Integrating albuminuria and GFR in the assessment of diabetic nephropathy. Nat. Rev. Nephrol. 5, 397-406 (2009)

8. W.C. Lee, H.C. Chen, C.Y. Wang et al., Cilostazol ameliorates nephropathy in type 1 diabetic rats involving improvement in oxidative stress and regulation of TGF-Beta and NF-kappaB. Biosci. Biotechnol. Biochem. 74, 1355-1361 (2010)

9. C.G. Schnackenberg, Am. J. Physiol. Regul. Integr. Comp. Phy- siol. 282, R335-R342 (2002)

10. A.S. Baldwin Jr, Annu. Rev. Immunol. 14, 649-683 (1996)

11. H. Ha, M.R. Yu, Y.J. Choi, M. Kitamura, H.B. Lee, J. Am. Soc. Nephrol. 13, 894-902 (2002)

12. F. Chiarelli, F. Cipollone, A. Mohn et al., Circulating monocyte chemoattractant protein-1 and early development of nephropathy in type 1 diabetes. Diabetes Care 25, 1829-1834 (2002)

13. K. Tashiro, I. Koyanagi, A. Saitoh et al., Urinary levels of monocyte chemoattractant protein-1 (MCP-1) and interleukin-8 (IL-8), and renal injuries in patients with type 2 diabetic nephropathy. J. Clin. Lab. Anal. 16, 1-4 (2002)

14. T. Morii, H. Fujita, T. Narita et al., Association of monocyte chemoattractant protein-1 (MCP-1) with renal tubular damage in diabetic nephropathy. J. Diabetes Complicat. 17, 11-15 (2003)

15. W.H. Tang, F.H. Lin, C.H. Lee et al., Cilostazol effectively attenuates deterioration of albuminuria in patients with type 2 diabetes: a randomized, placebo-controlled trial. Endocrine 45, 293-301 (2014)

16. L. Gao, F. Wang, B. Wang et al., Cilostazol protects diabetic rats from vascular inflammation via nuclear factor-kappa B-dependent downregulation of vascular cell adhesion molecule-1 expression. J. Pharmacol. Exp. Ther. 318, 53-58 (2006)

17. Y. Hattori, K. Suzuki, A. Tomizawa et al., Cilostazol inhibits cytokine-induced nuclear factor-kappa B activation via AMP activated protein kinase activation in vascular endothelial cells. Cardiovasc. Res. 81, 133-139 (2009)

18. J.S. Liu, T.J. Chuang, J.H. Chen et al., Cilostazol attenuates the severity of peripheral arterial occlusive disease in patients with type 2 diabetes: the role of plasma soluble receptor for advanced glycation end-products. Endocrine 49, 703-710 (2015)

19. X.M. Jiao, X.J. Jiao, X.G. Zhang et al., Cilostazol reduces microalbuminuria in type 2 diabetic nephropathy. Chin. Med. J. 126(22), 4395$4396(2013)$

20. J.M. Li, A.M. Shah, ROS generation by nonphagocytic NADPH oxidase; potential relevance in diabetic nephropathy. J. Am. Soc. Nephrol. 14(8 Suppl 3), S221-S226 (2003)

21. H. Ha, H.B. Lee, Reactive oxygen species and matrix remodeling in diabetic kidney. J. Am. Soc. Nephrol. 14(8 Suppl 3), S246-S249 (2003)

22. D.A. Antonetti, A.J. Barber, S.K. Bronson et al., Diabetic reti- nopathy: seeing beyond glucose-induced microvascular disease. Diabetes 55, 2401-2411 (2006)

23. J.W. Miller, A.P. Adamis, L.P. Aiello, Vascular endothelial growth factor in ocular neovascularization and proliferative dia- betic retinopathy. Diabetes Metab. Rev. 13, 37-50 (1997)

24. K.I. Jung, J.H. Kim, H.Y. Park, C.K. Park, Neuroprotective effects of cilostazol on retinal ganglion cell damage in diabetic rats. J. Pharmacol. Exp. Ther. 345, 457-463 (2013)

25. X. Zhang, S. Bao, B.D. Hambly, M.C. Gillies, Vascular endo- thelial growth factor-A: a multifunctional molecular player in 
diabetic retinopathy. Int. J. Biochem. Cell Biol. 41, 2368-2371 (2009)

26. R.L. Rosales, M.M. Santos, L.B. Mercado-Asis, Cilostazol: a pilot study on safety and clinical efficacy in neuropathies of diabetes mellitus type 2 (ASCEND). Angiology 62, 625-635 (2011) 
27. Pletal [package insert]. Rockville, MD: Otsuka America Phar- maceutical, Inc.; (2015)

28. Y. Aoki, M. Shimizu, N. Watanabe, The blood glucose level increased in parallel with the heart rate following cilostazol admin- istration in three diabetic patients. Intern. Med. 53, 859-863 (2014) 